\title{
La nouvelle offensive du Gouvernement contre la réglementation du travail
}

Sidnei Machado

\section{(2) OpenEdition}

1 Journals

Édition électronique

URL : https://journals.openedition.org/rdctss/1249

DOI : $10.4000 /$ rdctss. 1249

ISSN : 2262-9815

Éditeur

Centre de droit comparé du travail et de la sécurité sociale

\section{Édition imprimée}

Date de publication : 1 avril 2020

Pagination : 152-155

ISSN : 2117-4350

\section{Référence électronique}

Sidnei Machado, «La nouvelle offensive du Gouvernement contre la réglementation du travail », Revue de droit comparé du travail et de la sécurité sociale [En ligne], 1 | 2020, mis en ligne le 01 novembre 2021, consulté le 11 novembre 2021. URL : http://journals.openedition.org/rdctss/1249; DOI : https:// doi.org/10.4000/rdctss. 1249

\section{(c) (†) $९$}

Revue de droit comparé du travail et de la sécurité sociale est mise à disposition selon les termes de la Licence Creative Commons Attribution - Pas d'Utilisation Commerciale - Pas de Modification 4.0 International. 


\section{SIDNEI MACHADO}

UNIVERSITÉ FÉdÉRALE DE PARANÁ

\section{LA NOUVELLE OFFENSIVE DU GOUVERNEMENT CONTRE LA RÉGLEMENTATION DU TRAVAIL}

Le dialogue social au Brésil connaît actuellement des perturbations importantes. La crise économique très sérieuse, l'instabilité politique et les réformes de la législation du travail ont accentué la détérioration du marché du travail et affaibli les mécanismes institutionnels de protection des travailleurs.

Deux influences se font sentir. La première est due aux effets de la déréglementation accrue du travail depuis la réforme de 2017, avec la loi n¹3.467 en vigueur depuis le 11 novembre 2017. Contrairement à ce qu'espéraient ses partisans, cette réforme n'a entraîné aucune réduction du chômage. Bien au contraire, elle a mis en évidence un ensemble de facteurs qui nuisent autant au marché du travail qu'à la protection des travailleurs. Citons notamment une informalité croissante du marché du travail, un affaiblissement notable de l'action syndicale et de la négociation collective, et une restriction importante de l'accès des travailleurs à la justice. La deuxième conséquence, plus récente, découle de l'élection de Jair Bolsonaro à la Présidence de la République du Brésil en octobre 2018. L'arrivée au pouvoir de Bolsonaro, marquée par un discours libéral autoritaire, représente une nouvelle inflexion des politiques sociales, avec des propositions visant à généraliser les mesures ultralibérales, alimentées par une idéologie d'extrême-droite aux caractéristiques néo-fascistes qui menace de détruire les cadres institutionnels garantissant les droits sociaux du travail.

Cette contribution s'intéresse aux aspects fondamentaux du dispositif institutionnel du travail dans le contexte du Gouvernement de Jair Bolsonaro, et met en lumière les principales réformes législatives déjà mises en œuvre ainsi que les projets en cours de discussion au Parlement et dans l'opinion publique. II donne enfin un aperçu des contours nouveaux - et alarmants - de la politique et de la législation du travail actuellement engagées par le Gouvernement.

\section{LE PLAN DE RÉFORME DE BOLSONARO}

Le Président Jair Bolsonaro a été élu avec un programme libéral, un discours et des propositions autoritaires, et une stratégie de radicalisation singulière qui prend la forme d'une offensive déclarée contre les droits sociaux du travail. Depuis qu'il est en place, le Gouvernement a intensifié les attaques contre la reconnaissance et la protection des droits sociaux des travailleurs. Lorsque Bolsonaro a été élu, ses priorités ont ainsi porté sur l'approbation de son projet de réforme générale de la sécurité sociale brésilienne, et sur l'approfondissement des réformes visant à "moderniser les relations de travail », afin d'élargir la réforme de 2017 engagée par l'ancien Président Michel Temer (2016-2018).

Durant la campagne présidentielle de 2018, Jair Bolsonaro s'est appuyé sur l'argument selon lequel "moins de droits valent mieux que pas d'emplois ». L'une de ses rares propositions, lancée de manière générique, a consisté en la création d'une « carte de 
travail verte et jaune " (carteira de trabalho verde e amarela) en tant qu'option alternative et déréglementée aux contrats de travail. Dans la première interview donnée après son élection, il a ainsi rappelé que «Le Brésil a trop de droits. Il faut pousser plus loin la réforme du travail ", son Ministre de l'économie Paulo Guedes déclarant pour sa part que "les relations [de travail] au Brésil sont obsolètes et représentent des armes de destruction massive des emplois ».

\section{LA SUPPRESSION DU MINISTÈRE DU TRAVAIL}

La première mesure ayant eu un impact majeur sur le Gouvernement de Bolsonaro a été la suppression du ministère du Travail, de l'Emploi et de la Prévoyance (Ministério do Trabalho, Emprego e Previdência - MTE) le 7 janvier 20191. Créé en 1930, le ministère du Travail était chargé de réglementer et de superviser tous les aspects des relations de travail au Brésil. Certaines de ses activités ont ainsi été intégrées au ministère de l'Economie. L'élimination d'une institution qui servait de médiateur dans le conflit entre le capital et les travailleurs révèle une régression sociale et va fortement impacter les politiques publiques liées à l'emploi. En outre, cette mesure réduit l'efficacité des fonctions de contrôle de l'Etat brésilien.

\section{LE PROJET DE RÉFORME DE LA SÉCURITÉ SOCIALE}

Sous la pression des marchés, un projet attendu de réforme de la sécurité sociale brésilienne a été présenté à la Chambre des Députés le 20 février 2019, par le biais d'une proposition d'amendement à la Constitution (Proposta de Emenda à Constituição - PEC) $n^{\circ} 6 / 2019$. Approuvée ensuite par la Chambre, cette proposition pourrait provoquer un changement de paradigme dans le modèle brésilien de protection sociale, et avoir un impact énorme sur la société brésilienne. Plus généralement, ce projet de réforme des retraites vise à achever les précédentes réformes de marché amorcées après le coup d'Etat de 2016 par Michel Temer : I'une consistait en un amendement constitutionnel gelant les dépenses publiques pour une durée invraisemblable de 20 ans (amendement constitutionnel $n^{\circ} 95 / 2016$ ), et l'autre concernait la réforme du travail susmentionnée (loi $\left.n^{\circ} 13.467 / 2017\right)$. Ces trois réformes structurelles s'inscrivent dans la refonte et la redéfinition des politiques publiques.

Présenté par le Gouvernement de Bolsonaro comme la "nouvelle retraite ", le projet de réforme modifie le régime de protection sociale en reconfigurant la sécurité sociale des salariés du secteur privé, rural et urbain, ainsi que la retraite des fonctionnaires. Il englobe également les prestations sociales des personnes âgées, handicapées et démunies. La principale mesure proposée par cette réforme est l'introduction d'une condition d'âge minimum de 65 ans pour les hommes et de 62 ans pour les femmes, qui n'existait pas auparavant dans le système brésilien.

La réforme des retraites au Brésil est un projet très large, d'une grande complexité pour les politiques publiques, avec un impact important sur la protection sociale et sur les droits acquis des assurés (actuels et futurs). Elle englobe la sécurité sociale et les prestations sociales, et modifie le modèle de financement de la sécurité sociale dans les secteurs public et privé. D'un point de vue juridique, le projet de réforme soulève de nombreux

1 Mesure Provisoire, $n^{\circ} 870 / 2019$. 
problèmes, l'enjeu central étant la déconstitutionnalisation de l'ensemble des droits à la protection sociale prévus par la Constitution de 1988 et la réduction du rôle de l'Etat dans l'élaboration des politiques.

\section{LE TRAVAIL DU DIMANCHE}

En juin 2019, le Gouvernement de Bolsonaro a publié une loi ${ }^{2}$ qui étend la possibilité de travailler le dimanche à 6 secteurs de l'économie : les industries d'extraction d'huile végétale et de biodiesel, les industries du vin et des dérivés du raisin, les industries aérospatiales, le commerce en général, les établissements touristiques et les services de maintenance aérospatiale. Cette mesure a pour objectif d'accorder aux entreprises une plus grande liberté de production et d'emploi, y compris les dimanche et jours fériés. Le travail est donc désormais autorisé pour un grand nombre d'activités économiques dans lesquelles travailler le dimanche était jusqu'alors exceptionnel. Cependant, cette loi modifie et affaiblit le droit au repos hebdomadaire " de préférence le dimanche », prévu par la loi brésilienne depuis 1949 (loi n605) et figurant dans le texte même de la Constitution brésilienne de 1988 (article 7, XV).

\section{DES DÉROGATIONS AUX NORMES DE SANTÉ ET DE SÉCURITÉ AU TRAVAIL}

Dans le cadre de la réglementation de la santé et de la sécurité au travail, le Gouvernement du Président Bolsonaro a publié, en juillet 2019, un décret ayant pour objectif de «lever les obstacles bureaucratiques à l'entrepreneuriat ». Ce texte amorce la révocation et/ou la modification des 36 règles statutaires en vigueur, engendrant de ce fait l'assouplissement des règles de santé et de sécurité au travail. Naturellement, cette déréglementation inquiète les acteurs sociaux impliqués dans la promotion de la santé et de la sécurité au travail, qui craignent un accroissement des accidents du travail dans le pays, alors que ce dernier occupe déjà la $4{ }^{\text {ème }}$ place dans le monde en la matière.

\section{L'ASSOUPLISSEMENT DU CONTRÔLE DU TEMPS DE TRAVAIL}

La loi n¹3.874 sur le temps de travail a été publiée le 20 septembre 2019 dans le but de promouvoir la liberté économique et d'encourager l'esprit d'entreprise. Elle comprend des mesures visant à assouplir les horaires de travail dans les entreprises. En vertu de cette nouvelle loi, toutes les entreprises de moins de 20 salariés sont dispensées de tenir un registre des heures de travail réalisées. Un des autres mesures consiste en la création, par voie de convention individuelle ou collective, d'un mécanisme de comptabilisation des heures de travail exceptionnelles effectuées par le salarié : ces heures ne seront enregistrées que si la journée de travail du salarié dépasse la durée prévue par la loi ou par son contrat.

La restriction du contrôle du temps de travail aux seules entreprises de plus de 20 salariés, associée à un contrôle uniquement réalisé de façon exceptionnelle, constituent également des mesures d'assouplissement. Leur principale conséquence est cependant la possibilité de fraude, en cas de non-enregistrement des heures de travail réelles par l'employé, afin d'empêcher la rémunération des heures supplémentaires. Sans preuve des

2 Portaria n604, 18/06/2019. 
heures de travail enregistrées, le salarié lésé ne dispose en effet que d'un accès limité à la justice pour demander réparation des dommages.

C'est dans ce contexte que, tout en subissant les conséquences de l'importante réforme du travail engagée en 2017, le Gouvernement de Jair Bolsonaro promeut des changements législatifs importants, visant à amplifier la déréglementation et la flexibilisation du marché du travail au Brésil. Dans son discours politique, le Gouvernement privilégie une liberté économique accrue et place les droits des travailleurs dans une position secondaire par rapport aux marchés. Les réformes de Bolsonaro favorisent une augmentation de la liberté et du pouvoir des entreprises. La dernière initiative du Gouvernement fut de créer, en août 2019, le Groupe de réflexion sur les études avancées du travail (Grupo de Altos Estudos do Trabalho - GAET) pour la « modernisation des relations de travail et l'avenir du travail dans le pays ». Ce groupe de travail a été conçu dans le but d'accentuer encore davantage la déréglementation du travail. Comme en témoigne l'ensemble des mesures et initiatives législatives ici présentées, le programme de réforme de Bolsonaro, soutenu par le secteur des grandes entreprises, prend essentiellement pour cible les droits des travailleurs, désormais considérés comme des obstacles à la libre entreprise. 